\title{
DETERMINANTES DE SPREADS SOBERANOS DURANTE LA RECIENTE CRISIS FINANCIERA: EL CASO EUROPEO*
}

\author{
Lisana B. Martinez \\ Mercedes Teruel ${ }^{* * * * *}$ \\ Antonio Terceño
}

* doi: 10.11144/Javeriana.cao29-53.dssd. Este artículo es producto de la investigación “Grupo Consolidado en Mercados y Análisis Financiero", financiado por la Universidad Rovira i Virgili desde 01-01-14 hasta 3112-16. El artículo se recibió 07-11-2013 y se aprobó el 10-10-2016. Sugerencia de citación: Martinez L.B; Teruel, M., y Terceño, A. (2016). Determinantes de spreads soberanos durante la reciente crisis financiera: el caso europeo. Cuadernos de Administración, 29 (53), 77-100. http://dx.doi.org/10.11144/Javeriana. cao29-53.dssd

** Doctora en Economía y Empresas de la Univesitat Rovira i Virgili, Reus, España, 2013. Investigadora Asistente. IIESS, Departamento de Economía, Universidad Nacional del Sur, CONICET. Universidad Provincial del Sudoeste, Bahía Blanca, Argentina.

Correo electrónico: lbmartinez@iiess-conicet.gob.ar

*** Doctora en Economía y Empresa de la Universitat Rovira i Virgili, Reus, España, 2006. Profesor Agregado de Economía de la Universitat Rovira i Virgili, Reus, España.

Correo electrónico: mercedes.teruel@urv.cat

**** Doctor en Ciencias Económicas y Empresariales de la Universidad de Barcelona, España, 1995. Catedrático de Universidad del Departamento de Gestión de Empresas, Facultad de Economía y Empresa de la Universitat Rovira i Virgili, Reus, España.

Correo electrónico: antonio.terceno@urv.cat 


\section{Determinantes de spreads soberanos durante la reciente crisis financiera: el caso europeo}

\section{RESUMEN}

En este artículo se identifican los principales determinantes de spreads soberanos considerando el bono alemán y de EEUU como benchmarks de referencia, para países de la Unión Europea y de la Unión Monetaria Europea, desde 2004 hasta 2011 . Se aplica la metodología econométrica de datos de panel con efectos fijos. Los resultados presentan altos ajustes ante los distintos benchmarks. La crisis financiera presenta mayor impacto en los países de la eurozona. Futuras líneas de investigación podrían contemplar un periodo de estudio más amplio, incluir más variables macroeconómicas y agrupar los países de acuerdo con la similitud de sus fundamentales para realizar un análisis más exhaustivo de los mercados de deuda.

Palabras claves: spreads de bonos soberanos, euro área, crisis financiera, datos de panel.

Código JEL: Go1, G15, E43

\section{Sovereign bond spreads determinants during the last financial crisis: The European case}

\section{Determinantes de spreads soberanos durante a crescente crise financeira: o caso europeu}

\section{Resumo}

Neste artigo, identificam-se os principais determinantes de spreads soberanos considerando o bônus alemão e estadunidense como benchmarks de referência para países da União Europeia e da União Monetária Europeia desde 2004 até 2011. Aplica-se a metodologia econométrica de dados de painel com efeitos fixos. Os resultados apresentam altos ajustes entre os diferentes benchmarks. A crise financeira apresenta maior impacto nos país da eurozona. Futuras linhas de pesquisa poderiam compreender um período de estudo mais amplo, incluir mais variáveis macroeconômicas e agrupar os países de acordo com a semelhança de suas fundamentais para realizar uma análise mais exaustiva dos mercados de dívida.

Palavras-chave: crise financeira, dados de painel, eurozona, spreads de bônus soberanos.

Classificação JEL: Go1, G15, E43 


\section{Introducción}

Las crisis financieras han sido estudiadas desde diversas perspectivas de acuerdo con sus principales determinantes, consecuencias y efecto contagio generado. A su vez, desde los inicios de la reciente crisis financiera ha aumentado el interés por el incremento de los spreads de deuda en los países europeos.

La crisis financiera ha modificado las percepciones de riesgo de los inversores y la diversificación de las carteras de inversión ha propagado el riesgo de mercado. Los mercados europeos se han convertido en inestables, poniendo en tela de juicio la propia moneda única. La extensión de la crisis financiera ha obligado al Banco Central Europeo a exigir rigurosas medidas fiscales a los países miembros y a implementar paquetes de rescate financiero a través de la inyección de grandes cantidades de dinero (Grammatikos y Vermeulen, 2011) para aquellas economías más afectadas por la crisis, como Grecia, Irlanda, Portugal y España. Estas economías han visto reducido su acceso a los mercados financieros, fruto del aumento de su riesgo reflejado en un incremento del rendimiento de los bonos exigido por el mercado. Tal como mencionan Barrios et al. (2009), Arghyrou y Kontonikas (2011) y Sgherri y Zoli (2009), entre 2007 y 2010 los spreads se han incrementado notablemente en relación al bono de referencia alemán para algunos países de la euro zona.

En el presente artículo destacamos diversas contribuciones importantes, en primer lugar, identificamos los determinantes que afectan los spreads de bonos soberanos de la muestra de países de la Unión Europea (UE), distinguiendo el impacto en el grupo de los pertenecientes a la Unión Monetaria Europea (UME).

Seguidamente, analizamos el impacto de la crisis financiera sobre los spreads soberanos considerado variables dummies que indican el comienzo de la crisis a partir de julio de 2007 por un lado, y desde septiembre de 2008 por otro, dada la existencia de diversas conjeturas en relación el inicio de la crisis financiera.

Además, hemos analizado los spreads respecto a dos benchmarks: el bono alemán y de EEUU, ambos a diez años de vencimiento y nominados en euro. El primero de ellos es comúnmente utilizado en la literatura como referente. No obstante, en este trabajo hemos considerado ambos benchmarks dado que el segundo de ellos corresponde al país en el cual tuvo origen la crisis financiera y por lo tanto esperamos distintos efectos. 
La estructura del artículo es la siguiente: en la primera sección presentamos una breve revisión de la literatura referente a los determinantes de spreads soberanos; en la segunda, exponemos los datos utilizados y las hipótesis formuladas. Continuamos con la tercera sección a presentar la metodología aplicada; en la cuarta sección presentamos los modelos econométricos y los resultados obtenidos mediante la aplicación de datos de panel. Finalmente, presentamos las conclusiones del estudio.

\section{Revisión de la literatura}

Durante la última crisis financiera la rentabilidad de los bonos estatales ha sido objeto de especial atención en los países de la UE dado los efectos heterogéneos producidos en los mercados financieros. Numerosos artículos se refieren a tres principales grupos de determinantes de los diferenciales de los bonos soberanos (Codogno et al., 2003; Geyer et al., 2004; Bernoth et al., 2004; Barrios et al., 2009; Bernoth y Erdogan, 2012; Manganelli y Wolswijk, 2009; Klepsch y Wollmershäuser, 2011; Terceño et al., 2015; Bianchi, 2016; entre otros).

El primero de estos determinantes es el denominado riesgo de crédito, que incluye el riesgo de impago (default), el riesgo de rebaja de categoría y el riesgo del diferencial de crédito. Durante la crisis, la deuda y el déficit se han incrementado y los gobiernos han presentado grandes dificultades para hacer frente a mayores deudas. Como consecuencia, la percepción de default del mercado ha ido incrementando, lo cual conlleva a una disminución en la calificación crediticia de las economías y un aumento en el riesgo de diferencial de crédito.

El segundo determinante considerado es la llamada prima de liquidez. Un mercado líquido permite a los inversores tomar decisiones en cualquier momento, por lo que el número de operaciones financieras realizadas debe ser considerable para determinar el tamaño y la profundidad del mercado y, por lo tanto, el nivel de liquidez. A su vez, si los costes de transacción son altos, los inversores exigirán mayores rendimientos.

El riesgo de liquidez y el riesgo de crédito están interconectados (Barrios et al., 2009; Arghyrou y Kontonikas, 2011). Por un lado, si un gobierno aumenta la oferta de bonos, la presión sobre la prima de liquidez disminuye. Por otro lado, un aumento en la oferta de bonos se asocia con un aumento de la deuda pública y el déficit, lo cual genera que la prima de riesgo de crédito se incremente. 
El tercer determinante de la prima de riesgo es la denominada aversión al riesgo, la cual está representada por la cantidad de riesgo que los inversores están dispuestos a asumir cuando invierten en los mercados financieros. Por lo tanto, un aumento en la percepción de riesgo de la economía genera mayores spreads. Además, tal y como mencionan Barrios et al. (2009), la combinación de alta aversión al riesgo y grandes déficits de cuenta corriente tienden a magnificar la incidencia de deterioro de las finanzas públicas en los spreads de rendimiento de los bonos soberanos. Del mismo modo, una visión común y predominante de la literatura sobre los índices de bonos soberanos europeos, es que los diferenciales son impulsados por un factor común a nivel mundial, representado por factores internacionales como la percepción del riesgo (Codogno et al., 2003; Geyer et al., 2004; Manganelli y Wolswijk, 2009; Sgherri y Zoli, 2009).

Codogno et al. (2003) analizan los diferenciales de rendimiento entre 1990-2002 y demuestran que el riesgo de default influye positivamente en la brecha entre los mercados líquidos y seguros. Señalan que el movimiento en los diferenciales de rendimiento se explica principalmente por cambios en los factores de riesgo global, mientras que los factores de liquidezjuegan un papel menor. Geyer et al. (2004) consideran que las variables macroeconómicas y de liquidez no tienen influencia significativa sobre los spreads. La principal conclusión que abordan es que el riesgo de crédito es un indicador importante del riesgo sistemático en los países de la UME. Por otro lado, Balli (2008) analiza la integración financiera junto con el rendimiento de los bonos soberanos, y concluye que para el mercado de bonos europeos los factores internacionales juegan un papel más importante que los factores internos, como el riesgo de default y riesgo de liquidez. A su vez, dicho autor sostiene que el mercado de bonos del euro aún no está totalmente integrado económicamente.

Además, Schuknecht et al. (2010) hallan que los spreads de rendimiento de deuda antes y durante la crisis pueden ser explicados teniendo en cuenta las variables que reflejen la prima de liquidez y la aversión al riesgo de default. También señalan la existencia de desequilibrios fiscales y el cambio en la aversión al riesgo general después del colapso de Lehman Brothers y el aumento sucesivo de spreads de los bonos de los países de la UME.

Sgherri y Zoli (2009) sostienen que la evolución de los spreads soberanos de los miembros de la zona euro presenta una tendencia temporal similar en relación al riesgo global y, a su vez, la evolución ha incrementado en la misma dirección que la sensibilidad de la deuda. Estos autores señalan que la liquidez de los mercados de bonos soberanos juega un papel importante, aunque limitado al explicar los spreads. 
A su vez, Aßmann y Boysen-Hogrefe (2009) concluyen que los riesgos de default y de liquidez pueden explicar la existencia de mayores spreads de los bonos soberanos. Además, señalan que ambos riesgos han aumentado durante la crisis, aunque el riesgo de liquidez tiene mayor importancia que el riesgo de default. En línea con estos resultados, Beber et al. (2009) confirman que los inversores persiguen la liquidez y no la calidad del crédito en momentos de tensión en el mercado.

Blot et al. (2016) consideran que la literatura referente a la deuda soberana y al riesgo de impago ha ido evolucionando. Schucknecht et al. (2009) consideran las reducciones fiscales y las variables macroeconómicas y la influencia de los ciclos de negocios en las economías (Grandes, 2007). Blot et al. (2016) analizan la influencia de las profecías autocumplidas y la existencia de información asimétrica como posibles determinantes.

Bernoth y Erdogan (2012) afirman que no sólo se debe considerar el cambio de las variables macroeconómicas, sino que también se debe tener en cuenta la variación del riesgo de crédito en el tiempo. Estos autores realizan un análisis de coeficientes variantes en un modelo no paramétrico en el marco de datos de panel con efectos fijos y concluyen que los diferenciales de rendimiento de la deuda se ven significativamente afectados por organizaciones internacionales y por factores de riesgo específicos de cada país, como la liquidez y la prima de riesgo de default.

Tal como es sabido, el impacto de la crisis financiera ha afectado profundamente a los mercados de bonos. Durante los periodos de inestabilidad los inversores aumentan su aversión al riesgo, y cambian su cartera de inversión por activos con mayor liquidez y calidad. Estos dos efectos se conocen como flight-to-liquidity y flight-to-quality (Vayanos, 2004; Beber et al., 2009).

Por lo tanto, el estudio de los principales determinantes de los diferenciales es relevante para los gobiernos, dado que son importantes indicadores de vulnerabilidad. En consecuencia, la identificación de los principales factores que determinan el nivel de los spreads permite diseñar políticas orientadas a fortalecer la visión que tienen los mercados financieros a través del canal de liquidez o a través de la disminución de los déficits fiscales. Costantini et al. (2014) sostienen que los determinantes a largo plazo de los diferenciales de bonos soberanos son relevantes para decidir en qué medida se necesitan intervenciones de política estructural para reducir los diferenciales de rendimiento de la deuda soberana y las emisiones en monedas correctas y la competitividad divergente entre los miembros de la UME, especialmente cuando se considera el precio del default. 


\section{Datos e hipótesis}

La base de datos utilizada es Datastream. Se reúne información de un total de 16 países europeos: Austria, Bélgica, Dinamarca, España, Finlandia, Francia, Grecia, Hungría, Irlanda, Italia, Países Bajos, Polonia, Portugal, República Checa, Reino Unido y Suecia. La selección obedece a la disposición de datos de todas las variables explicativas utilizadas en cada uno de los países.

Como principal indicador de la evolución de los rendimientos de bonos gubernamentales se ha utilizado el Government Bond Index (GBI) calculado por JPMorgan.

Dentro de las características generales de los GBI, se destacan: el cálculo diario, están compuestos por bonos regularmente comercializados, a tasa fija y bonos domésticos de países que ofrecen una oportunidad al inversor institucional internacional, y que poseen un mercado de deuda líquido; lo cual implica que sean estables, activos y con emisiones regulares y vencimientos que oscilan entre los siete y diez años. Además, estos son considerados de alta calidad y se identifican con las características de ser representativos, ofrecen certezas, fiabilidad y transparencia (JPMorgan, 2001). Los índices que elabora JPMorgan miden la performance del mercado de renta fija mundial en cualquier nivel de análisis. Estos índices comprenden sólo instrumentos líquidos y de esta forma son considerados como apropiados benchmarks para inversores internacionales.

Para la realización del análisis empírico de spreads soberanos de países de la Unión Europea, se considera el GBI tanto para el benchmark alemán como para el de Estados Unidos. Las características de dicho índice se adaptan a los requisitos necesarios para evaluar la evolución de los spreads europeos; dada la existencia del mismo índice para todos los países de la muestra y el cálculo del mismo durante el periodo temporal requerido, con frecuencia trimestral, en una misma moneda en común como es el euro.

Respecto al resto de las variables seleccionadas como explicativas de los spreads soberanos, se trabajó con series temporales para el periodo 2004-2011 con frecuencia trimestral, medido al final de cada trimestre. Se presentan ciertas excepciones respecto a la frecuencia general utilizada con la variable relación de intercambio para el caso de Francia, dado que no hay datos disponibles a partir de Q2 de 2009, por lo cual se supuso que este último valor se mantiene constante hasta el final de 2011. Otra irregularidad con esta misma variable se da para el caso de Austria, Grecia y Portugal, dado que la 
frecuencia ofrecida es anual, por lo cual consideramos que los valores de los dos años se distribuyen linealmente en cada trimestre.

Siguiendo el análisis realizado por Rowland y Torres (2004), en el cual destaca la importancia del principio de parsimonia respecto al uso de variables significativas en los modelos, hemos trabajado con las siguientes variables presentadas en la tabla 1. Las mismas se presentan de forma agrupada de acuerdo con los principales riesgos que implican, tal como hemos citado en el apartado anterior. Siguiendo la misma línea presentada en la revisión de la literatura, destacamos que las agrupaciones pueden ser reagrupadas, dado que una variable puede representar más de un riesgo. Por lo tanto la agrupación realizada es sólo para fines ejemplificativos y no determinantes de cada riesgo específico.

\section{Tabla 1}

Variables explicativas

\begin{tabular}{lll}
\hline \multicolumn{1}{c}{ Riesgo de liquidez } & \multicolumn{1}{c}{ Riesgo de Crédito } & \multicolumn{1}{c}{ Aversión al riesgo } \\
\hline Reservas internacionales & Déficit público & Índice de precio de acciones \\
M2 & Deuda pública & Desempleo \\
Términos de intercambio & Crecimiento del PIB & Inflación \\
\hline \multicolumn{3}{c}{ Crisis07-Crisis08 } \\
\hline
\end{tabular}

Fuente: elaboración propia. Ver en el anexo la descripción general de las variables utilizadas en los modelos econométricos.

Se consideraron dos variables dummies en representación de la influencia del comienzo de la crisis financiera en los mercados de deuda pública. La primera de ellas corresponde a la fecha del 15 de julio de 2007 (Crisis07) momento a partir del cual comienzan a conocerse los problemas financieros de grandes compañías del sector, como son: Bear Stearns, Countrywide, y America Home Mortgage. La segunda fecha considerada es el 15 de septiembre de 2008 (Crisis08), la cual representa el día en el cual Lehman Brothers se declaró en quiebra.

Los spreads soberanos europeos permanecieron relativamente estables y a un nivel bajo hasta julio de 2007, aunque el mayor incremento en los mismos tuvo lugar a partir de septiembre de 2008. De acuerdo con A $\beta$ mann y Boysen-Hogrefe (2009), a mediados de 2007 se inició la crisis financiera, aunque tuvo su punto más relevante en septiembre de 2008 ante el colapso de Lehman Brothers. Al final de ese año, los spreads crecieron abruptamente en relación al bono de referencia alemán. Grammatikos y Vermeulen (2012) analizan la trasmisión de la crisis financiera y soberana desde 2003 a 2010 a los distintos miembros de la UME. Confirman que el colapso de Lehman Brothers determinó 
un punto de inflexión en la evolución de los mercados. Resultados similares fueron obtenidos por Bariviera et al. (2012) en relación a la eficiencia informativa del mercado de bonos europeos. Por lo tanto, dada la existencia de dos fechas claves en relación al inicio de la crisis, hemos considerado ambas con el fin de contrastar el impacto de la crisis en los mercados europeos. Tomando cada una de ellas valor igual a 1 para aquellos datos pertenecientes al periodo posterior a la fecha de corte considerada y valor igual a 0 para los datos previos a la misma.

Los estadísticos descriptivos y la matriz de correlaciones (tablas 2 y 3 ) muestran parámetros razonables por lo que no se presentan problemas econométricos entre las variables explicativas seleccionadas.

Tabla 2

Estadísticos descriptivos $(n=492)$

\begin{tabular}{lrrrr}
\hline \multicolumn{1}{c}{ Variable } & \multicolumn{1}{c}{ Media } & \multicolumn{1}{c}{ Desv. Est. } & \multicolumn{1}{c}{ Min } & \multicolumn{1}{c}{ Max } \\
\hline Spread (Alemania) & 0,942 & 1,608 & $-0,574$ & 9,638 \\
Spread (EEUU) & 0,572 & 1,762 & $-1,399$ & 9,689 \\
IndPrAcc & $6.864,470$ & $8.070,903$ & 171,990 & $43.165,530$ \\
Inflación & 106,157 & 6,605 & 94,800 & 135,600 \\
Déficit & 3,426 & $-4,814$ & $-32,400$ & 5,200 \\
Deuda Pub & 63,055 & 26,505 & 24,682 & 160,917 \\
Desempleo & 0,052 & 0,691 & $-2,600$ & 3,450 \\
PIB & 0,004 & 0,034 & $-0,191$ & 0,131 \\
TOT & 98,276 & 4,839 & 76,900 & 107,400 \\
M2 & 2,357 & 1,336 & 0,020 & 5,186 \\
Reservas & 1,157 & 4,597 & 0,0004 & 29,523 \\
\hline
\end{tabular}

Fuente: elaboración propia.

Tabla 3

Matriz de correlaciones

\begin{tabular}{|c|c|c|c|c|c|c|c|c|}
\hline & IndPrAcc & Inflación & Déficit & Deuda Pub Desempleo & PIB & TOT & M2 & Reservas \\
\hline IndPrAcc & 1 & & & & & & & \\
\hline Inflación & $0,136^{*}$ & 1 & & & & & & \\
\hline Déficit & $-0,039$ & $-0,287^{*}$ & 1 & & & & & \\
\hline Deuda Pub & 0,300 * & 0,220 * & $-0,499 *$ & 1 & & & & \\
\hline
\end{tabular}




\begin{tabular}{|c|c|c|c|c|c|c|c|c|c|}
\hline & IndPrAcc & Inflación & Déficit & Deuda Pub & Desempleo & PIB & TOT & M2 & Reservas \\
\hline Desempleo & $-0,027$ & 0,149 * & $-0,240^{*}$ & 0,102 & 1 & & & & \\
\hline PIB & $-0,001$ & $-0,112$ & 0,078 & $-0,078$ & 0,092 & 1 & & & \\
\hline TOT & 0,054 & $-0,133^{*}$ & $0,161^{*}$ & $-0,063$ & $-0,038$ & 0,021 & 1 & & \\
\hline M2 & $-0,144^{*}$ & 0,072 & $-0,236^{*}$ & $0,164^{*}$ & 0,078 & $-0,039$ & $-0,344^{*}$ & 1 & \\
\hline Reservas & $-0,046$ & 0,089 & $-0,164^{*}$ & $-0,038$ & 0,002 & $-0,015$ & 0,114 & $-0,384^{*}$ & 1 \\
\hline
\end{tabular}

Fuente: elaboración propia. Nota: * significativo al $1 \%$.

A continuación se presentan las hipótesis respecto al comportamiento esperado de las variables explicativas incluidas en los modelos econométricos.

H1: El indice de precios tiene un efecto negativo sobre los spreads soberanos.

Es sobradamente conocido que el mercado de renta fija y de renta variable mantiene una relación inversa; si sube la rentabilidad de los bonos (se incrementa el spread) se produce un desplazamiento inversor de renta variable a renta fija, por lo que el precio de las acciones bajará. Además, si los inversores son adversos al riesgo, cambiarán sus activos a mercados más líquidos y seguros, como son los instrumentos soberanos. Estos movimientos inversos en los precios de los activos financieros justifican la relación negativa entre ambos precios de mercado. La variable se expresa en términos logarítmicos para analizar la elasticidad de la variable respecto al spreads de los bonos

H2: El índice de inflación presenta una relación positiva respecto los spreads soberanos.

La tasa de inflación es un indicador representativo de la estabilidad macroeconómica. Tal como mencionan Baldacci et al. (2008), una alta tasa de inflación podría ser consecuencia de la monetización del déficit fiscal y representar la necesidad de mayores tasas de interés, aumentando el coste del capital (Min et al., 1998).

H3: El déficit público muestra un efecto positivo sobre los spreads soberanos.

Las crisis financieras generan alteraciones económicas que aumentan los problemas de pago de las deudas contraídas. Por lo tanto, es esperable que a medida que aumenten el déficit público respecto al PIB, los spreads soberanos varíen en el mismo sentido (Eichengreen y Mody, 1998; Rowland y Torres, 2004; Bernoth et al., 2004; Schuknecht et al., 2010). 
H4: El ratio deuda pública respecto el PIB presenta una relación positiva sobre los spreads soberanos.

La variable deuda pública respecto el PIB tiene una influencia ampliamente reconocida (Min, 1998; Kamin y von Kelist, 1999; Arora y Cerisola, 2001; Codogno et al., 2003; Schuknecht et al., 2010; Maltritz, 2012). Un índice bajo de esta variable indica que la economía produce un gran número de bienes y servicios que le generan suficientes ganancias para pagar las deudas. Una mayor carga de la deuda significa un mayor riesgo de impago agravado por déficits públicos. Este escenario ha sido común en algunos países de la UE durante los últimos cuatro años, particularmente en miembros de la UME. Hay que destacar que el nivel de esta variable, está limitada por los criterios del Tratado de Maastricht que los miembros de la UME deberían cumplir.

H5: La tasa de crecimiento del desempleo muestra un efecto positivo sobre los spreads soberanos.

Desde la materialización de la crisis financiera, algunos países europeos han experimentado mayores tasas de desempleo debido a una disminución en la actividad económica y la inversión. En consecuencia, esta reducción ha incrementado tanto el déficit público como así también los spreads de bonos soberanos. De acuerdo con Sgherri y Zoli (2009), la tasa de desempleo es un factor clave y una consecuencia importante de los problemas financieros globales, como así también una de las variables que mejor refleja los periodos recesivos.

H6: La tasa de variación del PIB es inversa a la reacción de los spreads soberanos.

Una variable representativa de la evolución de la economía real es el crecimiento del PIB, la cual tiene un efecto significativo en el mercado financiero. Hemos introducido esta variable rezagada un periodo con el fin de tener en cuenta el impacto retardado de la tendencia económica.

H7: La variable términos de intercambio presenta una relación inversa respecto los spreads soberanos.

Se introduce también la variable relación de intercambio, como indicador de la evolución del sector externo (Ades et al., 2000; Baldacci et al., 2008; Maltritz, 2012), la cual repre- 
senta el precio de las exportaciones de un país en relación con sus importaciones. Una disminución en los términos de intercambio significa que el precio promedio de exportación disminuye en relación con el precio medio de las importaciones. Una disminución de la variable términos de intercambio implica un deterioro de la economía y por lo tanto un efecto significativo en el mercado financiero.

H8: El ratio M2/PIB presenta una relación inversa respecto el nivel de spreads.

La variable M2 en relación al PIB es un indicador de la masa monetaria comúnmente conocida respecto al PIB de un país (Reinhart et al., 1998). Si este ratio aumenta, nos estaría indicando una situación de mejora a nivel país, esta mejora ejercerá una mejora en los spreads soberados con una disminución de los mismos.

H9: La cantidad de reservas internacionales respecto al PIB del país muestra una relación inversa respecto los spreads soberanos.

La deuda externa contraída por los países tiene que ser afrontada con reservas internacionales, por lo cual, si el ratio de esta variable respecto al PIB es pequeño, las probabilidades de existencia de una crisis de liquidez y riesgo de impago aumentan (Dailamini et al., 2005; Rowland y Torres, 2004; Min, 1998). La importancia de esta variable podría ser relativa en nuestro análisis, en particular en los países de la UME dado que un alto porcentaje de las reservas de estos países están en su propia moneda.

H10-11: La crisis financiera implica un aumento de los spreads soberanos.

En este caso y al trabajar con variables dummies la hipótesis se comprobará siempre que la variable sea significativa, es decir, si la crisis ha influenciado la variable deberá ser significativa. Cabe decir, que la crisis financiera acabó deteriorando las variables macroeconómicas de cada país. Esta transmisión de la crisis puramente financiera hacia una crisis tipo macroeconómica puede provocar que el impacto de la crisis en el 2007 sea diferente al impacto en el año 2008. Por ello introducimos las dos variables dummy, que a la vez nos permitirá detectar la velocidad de transmisión de la crisis desde el país originador a la Unión Europea. 


\section{Metodología}

Siguiendo el objetivo del presente trabajo, de identificar los principales determinantes de los spreads soberanos, aplicamos la metodología de datos de panel con efectos fijos. Los datos de panel permiten observar a un país durante diversos periodos de tiempo y permiten controlar la heterogeneidad individual. Para el presente análisis se ha asumido que los países son heterogéneos, por lo que la aplicación de datos de panel nos permite controlar dicha característica. Este supuesto es plausible dado que las características intrínsecas de los países recogidos en la muestra son diversas. Además, los datos de panel pueden revelar dinámicas difíciles de detectar con datos de corte transversal. En este sentido, son importantes para determinar las relaciones inter-temporales y también dan más información y grados de libertad. A pesar de todas estas ventajas, la literatura empírica sobre los determinantes de spreads ha utilizado escasamente esta metodología. Sin embargo, algunos trabajos de interés que aplican datos de panel son: Lemmen y Goodhart (1999), Rowland y Torres (2004) y Barrios et al. (2009).

\section{Análisis empírico de datos de panel}

Los modelos utilizados para la estimación de datos de panel, respecto a cada benchmark, son los siguientes:

$$
\begin{aligned}
& (1 ; 4) \text { SPREAD }_{\text {it }}=\beta_{0}+\beta_{1} \text { IndPrAcc }_{i t}+\beta_{2} \text { Inflación }_{\text {it }}+\beta_{3} \text { Deficit }_{\text {it }} \\
& +\beta_{4} \text { DeudaPub }_{i t}+\beta_{5} \text { Desempleo }_{\text {it }}+\beta_{6} \text { PIB }_{i t}+\beta_{7} \text { TOT }_{\text {it }}+\beta_{8} \mathrm{ML}_{\text {it }}+\beta_{9} \text { Reservas }_{\text {it }} \\
& +\mu_{i}+\gamma_{t}+\varepsilon_{i t} \\
& (2 ; 5) \text { SPREAD }_{i t}=\beta_{0}+\beta_{1} \text { IndPrAcc }_{i t}+\beta_{2} \text { Inflación }_{i t}+\beta_{3} \text { Deficit }_{\text {it }} \\
& +\beta_{4} \text { DeudaPub }_{i t}+\beta_{5} \text { Desempleo }_{\text {it }}+\beta_{6} \text { PIB }_{i t}+\beta_{7} \text { TOT }_{i t}+\beta_{8} \mathrm{M}_{i t}+\beta_{9} \text { Reservas }_{i t} \\
& +\beta_{10}{\text { Crisis } 07_{i t}+\mu_{i}+\gamma_{t}+\varepsilon_{i t}} \\
& (3 ; 6) \text { SPREAD }_{i t}=\beta_{0}+\beta_{1} \text { IndPrAcc }_{\text {it }}+\beta_{2} \text { Inflación }_{\text {it }}+\beta_{3} \text { Deficit }_{\text {it }} \\
& +\beta_{4} \text { DeudaPub }_{\text {it }}+\beta_{5} \text { Desempleo }_{\text {it }}+\beta_{6} \text { PIB }_{\text {it }}+\beta_{7} \text { TOT }_{i t}+\beta_{8} \mathrm{Mz}_{\text {it }}+\beta_{9} \text { Reservas }_{\text {it }}
\end{aligned}
$$

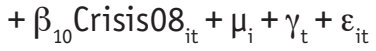

Donde $i \in\{1,2 \ldots, 16\}$ identifica el país " $i$ " y $t \in\{1, \ldots .31\}$ se refiere a las observaciones. La variable dependiente es el spread de bonos soberanos de cada país y $\beta_{i}$ son los coeficientes 
a estimar de cada variable. Por último, $\mu_{i^{\prime}} \gamma_{t^{\prime}}$ y $\varepsilon_{i t}$ representan los efectos de nivel individual de cada país, el efecto del tiempo y una perturbación aleatoria para la cual se asume que tiene media cero y varianza constante. Tanto el efecto individual como el efecto tiempo se presentan con el fin de controlar las características idiosincráticas de los países y los cambios estructurales que pueden haber afectado simultáneamente a todos los países.

Se controlaron los efectos individuales y temporales de las variables aplicando el Test de Haussman, a través del cual se verificó la hipótesis de utilizar efectos fijos.

La hipótesis nula se basa en la existencia de correlaciones significativas entre los regresores y los términos de error. Si dicha correlación no existe, entonces la aplicación de modelos de efectos aleatorios sería la más conveniente. Sin embargo, ante la existencia de correlaciones significativas el modelo de efectos fijos es el más adecuado.

Debemos mencionar además, que la muestra seleccionada es demasiado corta para analizar la existencia de patrones cointegrados. La heterogeneidad respecto a cada país y en cada periodo, está controlada por la estimación de los efectos fijos.

Con el fin de analizar la posible existencia de multicolinealidad, aplicamos el factor de inflación de varianza (VIF). Los resultados no muestras valores superiores a 10 (el mayor VIF es de 1,97), indicando que la presencia de multicolinealidad no es un problema.

\subsection{Spreads soberanos considerando el benchmark alemán}

En esta sección se presentan los resultados del análisis empírico respecto al índice de referencia alemán. Consideramos dos grupos, todos los países de la muestra y luego, sólo los miembros de la UME. Para cada grupo de países, realizamos tres regresiones.

Tal como puede observarse, todas las estimaciones presentan un buen ajuste y los signos de las variables explicativas consideradas corroboran las relaciones de causalidad establecidas en las hipótesis.

En términos generales, se destaca la significatividad de las variables Déficit Público, Deuda Pública y Desempleo como variables significativas para ambos grupos de países. La variable Inflación también se puede decir que es significativa en casi todas las estimaciones. 
En relación a las principales diferencias entre ambos grupos de países, respecto a la significatividad de variables específicas, se destaca que las variables índice de precio de acciones, términos de intercambio y reservas internacionales son significativas sólo para los países de la Unión Europea. Por otro lado, las variables crecimiento del PIB y el ratio M2 son significativas sólo para los países miembros de la Unión Monetaria Europea.

Con base en estas estimaciones iniciales, intentamos capturar el efecto de la crisis financiera en los spreads introduciendo las variables dummies. Tal como ha sido comentado previamente, utilizamos dos fechas como punto de referencia del comienzo de la crisis. Primero, en las estimaciones (2) y (5) incluimos la variable dummy Crisis 07, la cual reconoce el inicio de la crisis desde julio de 2007 en ambos grupos de países. Segundo, en las estimaciones (3) y (6) incluimos la variable dummy Crisis 08, la cual considera el auge de la crisis desde septiembre de 2008. El objetivo de incluir ambas variables se basa en analizar el posible efecto contagio de la crisis financiera iniciada en Estados Unidos hacia el resto de los países europeos.

Si consideramos las estimaciones correspondientes al grupo de países de la Unión Europea (estimaciones 2 y 3 ), podemos observar que las variables dummies resultaron ser no significativas. Lo cual nos estaría indicando que considerando todos los países de la muestra, pertenecientes a la UE, el efecto contagio de la crisis financiera no se ve claro. Este resultado se podría justificar por varias razones, entre ellas, la inclusión de países heterogéneos los cuales sufrieron shocks asimétricos en cuanto a intensidad y a ritmo del impacto de la crisis financiera. Otra explicación plausible es la transmisión de la crisis financiera hacia variables macroeconómicas, las cuales estarían recogiendo el impacto ejercido de forma temporal por la crisis financiera.

En este sentido, Kohonen (2014) analiza el incremento en los spreads soberanos en algunos países de la eurozona y encuentra que si bien la crisis financiera y el sucesivo efecto contagio fueron importantes en la alteración de los spreads, existen diferencias notables entre los distintos países de la región, los cuales sufrieron aumentos significativos en factores específicos de riesgo de cada país.

Sin embargo, al analizar solo los países miembros de la UME, ambas variables dummies resultaron ser significativas. 
Un aspecto relevante a destacar es la importancia del análisis respecto a este benchmark, dado que Alemania no sólo es el referente en cuestión, sino que también es miembro de la eurozona.

\section{Tabla 4}

Resultados de datos de panel, respecto al benchmark Alemán

\begin{tabular}{|c|c|c|c|c|c|c|}
\hline & \multicolumn{3}{|c|}{ Unión Europea (UE) } & \multicolumn{3}{|c|}{ Unión Monetaria Europea (UME) } \\
\hline & (1) & (2) & (3) & (4) & (5) & (6) \\
\hline \multirow{2}{*}{ IndPrAcc } & $-5,2 \mathrm{e}-5^{* * *}$ & $-5,4 \mathrm{e}-5^{* * *}$ & $-5,0 e-5^{* * *}$ & $-5,7 e-6$ & $-1,3 e-5$ & $-1,6 e-5$ \\
\hline & $(1,3 e-05)$ & $(1,3 e-5)$ & $(1,4 e-5)$ & $(1,72 e-5)$ & $(1,7 e-5)$ & $(1,8 \mathrm{e}-5)$ \\
\hline \multirow{2}{*}{ Inflación } & $0,0161^{*}$ & $0,0268 * *$ & 0,0137 & $0,0592 * * *$ & $0,105^{* * *}$ & $0,0758^{* * *}$ \\
\hline & $(0,0085)$ & $(0,0113)$ & $(0,0107)$ & $(0,0134)$ & $(0,0178)$ & $(0,0160)$ \\
\hline \multirow{2}{*}{ Déficit } & $0,0905^{* * *}$ & $0,0860 * * *$ & $0,0922^{* * *}$ & $0,117 * * *$ & $0,107^{* * *}$ & $0,110 * * *$ \\
\hline & $(0,0137)$ & $(0,0141)$ & $(0,0145)$ & $(0,0158)$ & $(0,0157)$ & $(0,0161)$ \\
\hline \multirow{2}{*}{ Deuda Pub } & $0,0916^{* * *}$ & $0,0901^{* * *}$ & $0,0914^{* * *}$ & $0,114^{* * *}$ & $0,111^{* * *}$ & $0,116^{* * *}$ \\
\hline & $(0,0050)$ & $(0,0050)$ & $(0,0050)$ & $(0,0057)$ & $(0,0056)$ & $(0,0058)$ \\
\hline \multirow{2}{*}{ Desempleo } & $0,167 * * *$ & $0,174 * * *$ & $0,165^{* * *}$ & $0,137^{* *}$ & $0,155^{* *}$ & $0,145^{* *}$ \\
\hline & $(0,0538)$ & $(0,0539)$ & $(0,0542)$ & $(0,0676)$ & $(0,0662)$ & $(0,0675)$ \\
\hline \multirow{2}{*}{ PIB } & $-1,271$ & $-1,275$ & $-1,257$ & $-5,593^{*}$ & $-6,266^{* *}$ & $-6,144^{*}$ \\
\hline & $(1,049)$ & $(1,047)$ & $(1,050)$ & $(3,130)$ & $(3,063)$ & $(3,130)$ \\
\hline \multirow{2}{*}{ ТОТ } & $-0,0297^{*}$ & $-0,0317^{* *}$ & $-0,0310^{* *}$ & $-0,0230$ & $-0,0233$ & $-0,0163$ \\
\hline & $(0,0152)$ & $(0,0152)$ & $(0,0156)$ & $(0,0196)$ & $(0,0191)$ & $(0,0198)$ \\
\hline \multirow{2}{*}{ M2 } & $-0,159$ & $-0,0819$ & $-0,175$ & $-0,975^{* * *}$ & $-0,808^{* * *}$ & $-0,919 * * *$ \\
\hline & $(0,195)$ & $(0,202)$ & $(0,199)$ & $(0,230)$ & $(0,229)$ & $(0,231)$ \\
\hline \multirow{2}{*}{ Reservas } & $-0,247 * * *$ & $-0,247 * * *$ & $-0,248 * * *$ & $-10,15$ & $-10,28$ & $-7,330$ \\
\hline & $(0,0288)$ & $(0,0288)$ & $(0,0289)$ & $(8,840)$ & $(8,638)$ & $(8,930)$ \\
\hline \multirow{2}{*}{ Crisis 07} & & $-0,198$ & & & $-0,670^{* * *}$ & \\
\hline & & $(0,136)$ & & & $(0,175)$ & \\
\hline \multirow{2}{*}{ Crisis 08} & & & 0,0563 & & & $-0,345^{*}$ \\
\hline & & & $(0,148)$ & & & $(0,184)$ \\
\hline \multirow{2}{*}{ Constant } & $-2,308$ & $-3,231^{*}$ & $-1,903$ & $-8,270^{* * *}$ & $-12,98^{* * *}$ & $-10,83^{* * *}$ \\
\hline & $(1,785)$ & $(1,893)$ & $(2,082)$ & $(2,647)$ & $(2,863)$ & $(2,969)$ \\
\hline \multirow{2}{*}{ Hausman test } & 231,93 & 202,06 & 227,67 & 383,48 & 381,83 & 384,41 \\
\hline & 0,0000 & 0,0000 & 0,0000 & 0,0000 & 0,0000 & 0,0000 \\
\hline Observaciones & 494 & 494 & 494 & 308 & 308 & 308 \\
\hline
\end{tabular}




\begin{tabular}{lcccccc}
\hline & \multicolumn{3}{c}{ Unión Europea (UE) } & \multicolumn{3}{c}{ Unión Monetaria Europea (UME) } \\
\cline { 2 - 7 } & $(1)$ & $(2)$ & $(3)$ & $(4)$ & $(5)$ & $(6)$ \\
\hline $\mathrm{R}^{2}$ & 0,607 & 0,609 & 0,607 & 0,723 & 0,737 & 0,727 \\
\hline Países & 16 & 16 & 16 & 10 & 10 & 10 \\
\hline
\end{tabular}

Nota: errores estándar entre paréntesis. *** Significativos at 1\%, ** al 5\%,y * al $10 \%$.

Fuente: elaboración propia.

La principal conclusión parcial de este apartado es que los spreads de los bonos soberanos son parcialmente explicados por el déficit público, la deuda pública, el desempleo e inflación. A su vez, se vislumbran interesantes diferencias entre los miembros de la UME y los que no pertenecen a la zona euro.

En general, las estimaciones presentan un buen nivel de ajuste. Por lo tanto, se puede decir que se han incluido variables explicativas relevantes.

\subsection{Spreads soberanos considerando el benchmark de Estados Unidos}

En esta sección presentamos un análisis similar al de la sección anterior pero considerando como bono de referencia para el cálculo de los spreads el índice de bono a diez años de EEUU. En la tabla 5 se vislumbra que ambos grupos de países presentan más variables significativas en común. Se destaca la significatividad de las variables: Índice precios de las acciones, inflación, déficit público, deuda pública, desempleo, PIB y TOT.

Las principales diferencias entre ambos grupos de países analizados se destacan ante la inclusión de las variables M2, reservas internacionales y las variables dummies crisis 07 y crisis 08 .

Respecto a la variable M2, representativa de la masa monetaria respecto al PIB de cada país, podemos observar que la misma presenta un comportamiento similar a los análisis realizados considerando el benchmark alemán. Siendo significativa y con signo negativo para los miembros de la euro zona. Este resultado indica que a medida que aumenta la masa monetaria respecto al PIB, los spreads soberanos han de disminuir.

Por otro lado y de forma similar a los resultados obtenidos respecto a los modelos considerando el benchmark alemán, es el análisis correspondiente a la variable Reservas Internacionales, la cual es significativa y de signo negativo para los países miembros de 
la UE y no para los de la UME. Este resultado, posiblemente pueda estar relacionado con las condiciones que deben cumplir los miembros de la UME en relación a las reservas que respalden la moneda común.

En relación con las variables dummies, representativas de periodos distintos en cuanto al inicio de la crisis financiera, los resultados son un tanto ambiguos. Respecto a la incidencia desde el 2007, podemos observar que el efecto de la variable dummy es significativo para ambos grupos de países.

Sin embargo, al considerar el efecto de la crisis desde el año 2008, esta variable es no significativa para los países de la UE, pero sí para los miembros de la UME, aunque en este caso el signo de la variable es negativo. La explicación del cambio de signo entre el periodo 2007 y el periodo 2008 puede ser el hecho que la crisis financiera se transmitiese hacia los principales agregados macroeconómicos y sean estos los que ejercen presión respecto los spreads. Por lo tanto, el impacto del shock financiero sólo ejerce un efecto sobre el alza de los spreads durante el inicio de la crisis, mientras que este queda reflejado sobre una mayor sensibilidad de las variables macroeconómicas.

La crisis de hipotecas subprime no fue un acontecimiento financiero más en la historia económica; no sólo por haberse originado en una economía con fuertes vínculos económicos con el resto del mundo, sino también por haberse producido en un sector particular, donde las nuevas formas de globalización, las diferentes preferencias de los inversores respecto al riesgo y por ende la diversificación en las carteras de inversión ayudaron a propagar los riesgos del mercado hacia el resto del mundo, afectando fundamentalmente a la Unión Monetaria Europea, evidenciado en un primer instante por la depreciación del euro.

A partir de entonces, aquellas economías europeas más débiles se desestabilizaron financiera y macroeconómicamente, reflejándose dichas inestabilidades a través de las altas primas de riesgo de las nuevas emisiones de deuda. De acuerdo con el análisis realizado, a pesar de la convergencia entre los rendimientos de los bonos de los países que integran la euro zona las diferencias continúan existiendo dado que los mercados no son perfectamente homogéneos y por lo tanto la vulnerabilidad de los mismos a las crisis financieras e impactos económicos es diferente. Este hecho ha quedado claramente contrastado con la reciente crisis financiera y, además, pone de relieve que aunque exista una moneda única, esto no es suficiente para hablar de un sector financiero único, ni tan si quiera de un mercado de bonos. 


\section{Tabla 5}

Resultados de datos de panel para miembros de la UE y UME, respecto al benchmak de EEUU

\begin{tabular}{|c|c|c|c|c|c|c|}
\hline \multirow{2}{*}{ Variables } & \multicolumn{3}{|c|}{ Unión Europea (EU) } & \multicolumn{3}{|c|}{ Unión Monetaria Europea (UME) } \\
\hline & (1) & $(2)$ & (3) & (4) & (5) & $(6)$ \\
\hline \multirow{2}{*}{ IndPrAcc } & $-9,1 e-5^{* * *}$ & $-8,0 \mathrm{e}-5^{* * *}$ & $-9,6 e-5^{* * *}$ & $-3,9 e-5^{* *}$ & $-3,4 e-5^{*}$ & $-5,6 e-5^{* * *}$ \\
\hline & $(1,5 e-5)$ & $(1,4 e-5)$ & $(1,6 e-5)$ & $(1,9 e-5)$ & $(1,9 e-5)$ & $(1,9 e-5)$ \\
\hline \multirow{2}{*}{ Inflación } & $0,0557 * * *$ & 0,00139 & $0,0624 * * *$ & $0,0994^{* * *}$ & $0,0595^{* * *}$ & $0,126^{* * *}$ \\
\hline & $(0,0096)$ & $(0,0122)$ & $(0,0121)$ & $(0,0147)$ & $(0,0197)$ & $(0,0174)$ \\
\hline \multirow{2}{*}{ Déficit } & $0,0738 * * *$ & $0,0965 * * *$ & $0,0691 * * *$ & $0,107 * * *$ & $0,115^{* * *}$ & $0,0969 * * *$ \\
\hline & $(0,0155)$ & $(0,0152)$ & $(0,0163)$ & $(0,0173)$ & $(0,0173)$ & $(0,0175)$ \\
\hline \multirow{2}{*}{ Deuda Pub } & $0,0852 * * *$ & $0,0926 * * *$ & $0,0855^{* * *}$ & $0,109 * * *$ & $0,112 * * *$ & $0,113 * * *$ \\
\hline & $(0,0056)$ & $(0,0055)$ & $(0,0056)$ & $(0,0062)$ & $(0,0062)$ & $(0,0063)$ \\
\hline \multirow{2}{*}{ Desempleo } & $0,249 * * *$ & $0,218^{* * *}$ & $0,254 * * *$ & $0,228^{* * *}$ & $0,213 * * *$ & $0,241 * * *$ \\
\hline & $(0,0608)$ & $(0,0582)$ & $(0,0611)$ & $(0,0739)$ & $(0,0731)$ & $(0,0733)$ \\
\hline \multirow{2}{*}{ PIB } & $-2,007^{*}$ & $-1,987^{*}$ & $-2,046^{*}$ & $-8,113^{* *}$ & $-7,531^{* *}$ & $-8,986 * * *$ \\
\hline & $(1,184)$ & $(1,131)$ & $(1,185)$ & $(3,423)$ & $(3,382)$ & $(3,400)$ \\
\hline \multirow{2}{*}{ TOT } & $-0,0490$ *** & $-0,0388^{* *}$ & $-0,0455^{* * *}$ & $-0,0440$ ** & $-0,0437^{* *}$ & $-0,0335$ \\
\hline & $(0,0171)$ & $(0,0164)$ & $(0,0176)$ & $(0,0214)$ & $(0,0211)$ & $(0,0215)$ \\
\hline \multirow{2}{*}{ M2 } & $-0,219$ & $-0,612^{* * *}$ & $-0,177$ & $-1,046 * * *$ & $-1,190$ *** & $-0,957^{* * *}$ \\
\hline & $(0,220)$ & $(0,218)$ & $(0,225)$ & $(0,252)$ & $(0,253)$ & $(0,251)$ \\
\hline \multirow{2}{*}{ Reservas } & $-0,257^{* * *}$ & $-0,258 * * *$ & $-0,256^{* * *}$ & $-3,412$ & $-3,295$ & 1,045 \\
\hline & $(0,0325)$ & $(0,0311)$ & $(0,0326)$ & $(9,667)$ & $(9,536)$ & $(9,700)$ \\
\hline \multirow{2}{*}{ Crisis 07} & & $1,001^{* * *}$ & & & $0,579 * * *$ & \\
\hline & & $(0,147)$ & & & $(0,193)$ & \\
\hline \multirow{2}{*}{ Crisis 08} & & & $-0,154$ & & & $-0,546^{* * *}$ \\
\hline & & & $(0,167)$ & & & $(0,200)$ \\
\hline \multirow{2}{*}{ Constant } & $-4,209 * *$ & 0,459 & $-5,321 * *$ & $-10,23 * * *$ & $-6,158^{*}$ & $-14,28 * * *$ \\
\hline & $(2,016)$ & $(2,044)$ & $(2,349)$ & $(2,895)$ & $(3,161)$ & $(3,225)$ \\
\hline \multirow{2}{*}{ Hausman test } & 96,81 & 163,24 & 97,69 & 0,00 & 0,00 & 0,00 \\
\hline & 0,0000 & 0,0000 & 0,0000 & - & - & - \\
\hline Observaciones & 494 & 494 & 494 & 308 & 308 & 308 \\
\hline $\mathrm{R}^{2}$ & 0,634 & 0,667 & 0,635 & 0,742 & 0,750 & 0,748 \\
\hline Países & 16 & 16 & 16 & 10 & 10 & 10 \\
\hline
\end{tabular}

Nota: Errores estándar entre paréntesis. *** Significativos at 1\%, ** al 5\%,y * al $10 \%$.

Fuente: elaboración propia. 


\section{Conclusiones}

A lo largo de este trabajo analizamos, bajo distintas alternativas, la incidencia de un conjunto de variables en la determinación de los spreads soberanos. En primer lugar, realizamos diversas estimaciones respecto al referente más conocido como es el índice de bonos alemán. Seguidamente, consideramos dos grupos de países, aquellos que pertenecen a la UE y los de la UME.

Se pueden destacar un conjunto de variables significativas comunes a ambos grupos de países, tales como el déficit público, la deuda pública y el desempleo. A su vez, se identifican variables que son significativas para los miembros de la eurozona y no para el resto de los países de la UE y viceversa.

Siguiendo con el objetivo propuesto, se consideraron variables dummies a fin de evaluar la incidencia de la crisis financiera, mediante las cuales se observa un mayor efecto en los países miembros de la UME, siendo significativas las mismas en el 2007 y en el 2008. Consideramos que estos resultados responden a la existencia de países heterogéneos de la muestra y en la absorción del impacto de la crisis financiera por las variables macroeconómicas explicativas de los determinantes soberanos.

Por otro lado, hemos replicado los modelos previos considerando como benchmark el índice de bonos de EEUU. En este caso, se observó que ambos grupos de países presentan mayor cantidad de variables significativas en común, presentando las variables M2 y reservas internacionales significatividades diferentes en cada grupo, al igual que en el caso de las regresiones respecto al benchmark alemán.

En este análisis el efecto de la crisis financiera es significativo. El vínculo que podemos establecer para estas estimaciones se relaciona con la presencia de un índice de bonos de referente correspondiente al país en el cual tuvo origen el inicio de la crisis, a partir del 2007.

Al considerar el bono de EEUU como benchmark, el ajuste de los modelos es mayor para todas las estimaciones. Este resultado es interesante dado que la economía norteamericana ha sido el epicentro de la crisis financiera y a su vez, debemos recordar que el referente comúnmente utilizado por los países europeos es el bono alemán. 
Las estimaciones para los países de la UME muestra un mejor ajuste que los de de la UE. Este efecto refleja la influencia de la incidencia de la moneda común. A pesar del efecto de la crisis financiera sobre estas economías y las distintas situaciones económicas de los miembros de la UME durante los últimos años, la presencia de una moneda común hace que las variables explicativas de este grupo sean más significativas.

Una posible explicación para este incremento en los niveles de significatividad es que la moneda única hace a los países miembros de la UME más homogéneos (respecto a los mercados financieros) y favorece un mayor efecto contagio que entre los miembros de la UE.

Destacamos dos aspectos importantes respecto a los spreads soberanos: primero, que la última crisis financiera resaltó la importancia de los factores domésticos respecto a la incidencia en los spreads de deuda, los cuales estaban latentes en el periodo previo al inicio de la crisis. En el mismo sentido, la crisis evidenció la heterogeneidad de los países de la UME reflejada en sus mercados de renta fija. Dichos mercados, antes de la crisis mantenían un comportamiento razonablemente homogéneo, pero con la llegada de la crisis esta homogeneidad quedo rota. Segundo, la selección del benchmark es muy importante y puede hacer el modelo más explicativo, además en nuestro análisis resulta destacable el hecho de que utilizando el benchmark estadounidense los modelos resulten más explicativos de la evolución del spread que utilizando el benchmark alemán.

Como futuras líneas de investigación planteamos la incorporación de un período de estudio más amplio, que permita evaluar si el efecto contagio de la crisis financiera se disipó o dejó a los mercados de deuda soberana alterados. A su vez, la utilización de un mayor número de variables macroeconómicas mediante la aplicación de otras metodologías, que permitan agrupar los países de acuerdo con la similitud de sus fundamentals contribuirá a realizar un análisis más exhaustivo de los mercados de deuda seleccionados.

\section{Referencias}

Ades, A., Kaune, F., Leme, P., Masih, R., Tenengauzer, D. (2000). Introduction GS-ESS: A new framework for assessing fair value in Emerging Markets hard-currency debt. Global Economics Paper, 45, 1-25.

Arghyrou M.G., and Kontonikas, A. (2011). The EMU Sovereign-debt crisis: Fundamentals, expectations and contagion. European Economy, 436, 1725-3187.

Arora, V., and Cerisola, M. (2001). How does U.S. monetary policy influence sovereign spreads in emerging markets? IMF Staff Papers, 48 (3), 474-498. 
A $\beta$ mann, C., and Boysen-Hogrefe J. (2009). Determinants of government bond spreads in the euro area - in good times as in bad. Kiel Institute for the World Economy, Working Paper 1548.

Baldacci, E., Gupta, S., and Mati, A. (2008). Is (still) mostly fiscal? Determinants of sovereign spreads in emerging markets. IMF Working Papers 08/259, 1-23.

Bariviera, AF, Guercio, MB, and Martinez, LB. (2012). A comparative analysis of the informational efficiency of the fixed income market in seven European countries. Economics Letters, 116 (3), 426-428.

Barrios, S., Iversen, P., Lewyowska, M., and Setze, R. (2009). Determinants of intra-euro area government bond spreads during the financial crisis. European Economy, 388, 1-26.

Beber, A., Bryt, M., and Kavajecz, K. (2009). Flight-to-quality or flight-to-liquidity? Evidence from the euro-area bond market. Review of Financial Studies, 22, 925-957.

Bernoth, K., von Hagen J., and Schuknecht, L. (2004). Sovereign risk premiums in the European government bond market. European Central Bank, Working Paper 369, 1-39.

Bernoth, K., and Erdogan, B. (2012). Sovereign bond yield spreads: A time varying coefficient approach, Journal of International Money and Finance, 31, 639-656.

Bianchi (2016). Sovereign risk premia and the international balance sheet: Lessons from the European Crisis. Open Economic Review, 27, 471-493.

Blot, C., Ducoudré, B., and Timbeau, X. (2016). Sovereign debt spread and default in a model with self-fulfilling prophecies and asymmetric information. Journal of Macroeconomics, 47, 281-299.

Codogno, L., Favero, C., and Missale, A. (2003). Yield spreads on EMU government bonds. Economic Policy, October, 503-532.

Costantini, M., Fragetta, M., and Giovanni, M. (2014). Determinants of sovereign bond yield spreads in the EMU: An optimal currency area perspective. European Economic Review, 70, 337-349.

Dailami, M., Masson, P.R., and Padou, J.J. (2005). Global monetary conditions versus country-specific factors in the determination of emerging market debt spreads. The World Bank Policy Research, 3626, 1-31.

Eichengreen, B., and Mody, A. (1998). What explains changing spreads on emerging markets debt: Fundamentals or market sentiment? NBER Working Paper, 6408, 1-45.

Fatih, Ö., Erdal, Ö., and Gülbin, Ş. (2009). Emerging market sovereign spreads global financial conditions y US macroeconomic news. Economic Modelling , 26, 526-531.

Geyer, A., Kossmeier, S., and Pichler, S. (2004). Measuring systematic risk in EMU spreads, Review of Finance, 8 (2), 171-197.

Gómez-Puig, M. (2009). Systemic and idiosyncratic risk in EU-15 Sovereign yield spreads after seven years of Monetary Union. European Financial Management, 15, 971-1000. 
Grammatikos, T., and Vermeulen, R. (2011). Transmission of the financial sovereign debt crises to the EMU: Stock Prices, CDS spreads and Exchange Rates. Journal of International Money and Finance, 31 (3), 517-533.

Grandes, M. (2007). The determinants of sovereign bond spreads: Theory and facts from Latin America, Cuadernos de Economía, 44, 151-181.

JPMorgan (2001). Government Bond Index Technical Document.

Kamin, S.B., and von Kleist, K. (1999). The evolution and determinants of emerging market credit spreads in the 1990s. BIS Working Papers, 68, 1-34.

Kaminsky, G., and Schmukler, S. (2002). Emerging market instability: Do sovereign ratings affect country risk and stock returns? The World Bank Economic Review, 16 (2), 171-195.

Klepsch, C., and Wollmershauser, T. (2011). Yield spreads on EMU government bonds. How the financial crisis has helped investors to rediscover risk. Intereconomics, 46 (3), 169-176.

Kohonen, A. (2014). Transmission of government default risk in the Eurozone. Journal of International Money and Finance, 47, 71-85.

Lemmen, J.J., and Goodhart, C.A. (1999). Credit risks y European government bond markets: A panel data econometric analysis, Eastern Economic Journal, 25 (1), 77-105.

Maltritz, D. (2012). Determinants of sovereign yield spreads in the Eurozone: A Bayesian approach, Journal of International Money and Finance, 31, 657-672.

Manganelli, S., and Wolswijk, G. (2009). What drives spreads in the Euro area government bond market? Economic Policy, April, 193-240.

Min, H. (1998). Determinants of emerging market bond spread. Do economic fundamentals matter?, Policy Research Working Paper, 1899, 1-31.

Pagano, M. (2004). The European bond markets under EMU, Oxford Review of Economic Policy, $20(4), 531-554$.

Reinhart, C., Kaminsky, G, and Lizondo, S. (1998). Leading indicators of currency crises, International Monetary Fund, 45 (1), 1-48.

Rowland, P., and Torres, J.L. (2004). Determinants of spread and creditworthiness for emerging market sovereign debt: A panel data study, Banco de la República, Borradores de Economía, 295, 1-55.

Schucknecht, L., von Hagen, J., and Wolswijk, G. (2009). Government risk premiums in the bond market: EMU and Canada. European Journal of Political Economy, 25, 371-384.

Schuknecht, L., von Hagen, J., and Wolswijk, G. (2010). Government bond risk premiums in the EU revisited: The impact of the financial crisis. European Central Bank. Working paper, 1152, 1-27.

Sgherri S., Zoli E. (2009). Euro area sovereign risk during the crisis, IMF Working Paper/09/222, $1-22$. 
Terceño-Gómez, A., Martinez, L.B., Sorrosal-Forradellas, M., and Guercio, M.B. (2015). Sovereign bond spreads and economic variables of European Countries under the analysis of self-organizing maps, Scientific Methods for the Treatment of Uncertainty in Social Sciences 377 of the series Advances in Intelligent Systems and Computing, № 377, 347-358.

Vayanos D. (2004). Flight to quality, flight to liquidity, and the pricing of risk. NBER- Working Papers 10327, 1-54.

Von Hagen, J, Schuknecht, L, and Wolswijk, G. (2011). Government bond risk premiums in the EU revisited: The impact of the financial crisis. European Journal of Political Economy, $24,36-43$.

\section{Anexo}

A continuación se presenta la descripción general de las variables utilizadas en los modelos econométricos.

IndPrAcc: Índice precios de las acciones. Se consideró la evolución del índice de acciones más reconocido de cada país.

Inflación: Índice de inflación. Índice elaborado por el Fondo Monetario Internacional. Déficit: Déficit Público sobre PIB. Ratio calculado por el FMI.

Deuda Pub: Deuda Pública sobre PIB.

Desempleo: Crecimiento del desempleo. Expresado en porcentaje.

PIB: Crecimiento PIB

TOT: Términos de intercambio. Expresado en porcentaje como ratio del precio de exportaciones sobre precio de importaciones.

M2: M2 sobre PIB

Reservas: Reservas internacionales sobre PIB

Crisis07 y Crisis08: (variables dummies) 